Methodology article

\title{
Interfering ribonucleic acids that suppress expression of multiple unrelated genes
}

\author{
Toby Passioura*, Mary M Gozar, Amber Goodchild, Andrew King, \\ Greg M Arndt, Michael Poidinger, Donald J Birkett and Laurent P Rivory
}

Address: Johnson \& Johnson Research Pty Ltd, Sydney, Australia

Email: Toby Passioura* - toby.passioura@gmail.com; Mary M Gozar - mary_gozar@yahoo.com; Amber Goodchild - burns_amber@lilly.com; Andrew King - aking4208@gmail.com; Greg M Arndt - greg.arndt@bigpond.com; Michael Poidinger - poidingermi@lilly.com;

Donald J Birkett - birkprof@hdbirkett.com; Laurent P Rivory - l.rivory@unsw.edu.au

* Corresponding author

Published: 16 June 2009

BMC Biotechnology 2009, 9:57 doi:10.1186/1472-6750-9-57
Received: 19 November 2008

Accepted: 16 June 2009

This article is available from: http://www.biomedcentral.com/I472-6750/9/57

(C) 2009 Passioura et al; licensee BioMed Central Ltd.

This is an Open Access article distributed under the terms of the Creative Commons Attribution License (http://creativecommons.org/licenses/by/2.0), which permits unrestricted use, distribution, and reproduction in any medium, provided the original work is properly cited.

\begin{abstract}
Background: Short interfering RNAs (siRNAs) have become the research tool of choice for gene suppression, with human clinical trials ongoing. The emphasis so far in siRNA therapeutics has been the design of one siRNA with complete complementarity to the intended target. However, there is a need for multi-targeting interfering RNA in diseases in which multiple gene products are of importance. We have investigated the possibility of using a single short synthetic duplex RNA to suppress the expression of VEGF-A and ICAM-I; genes implicated in the progression of ocular neovascular diseases such as diabetic retinopathy.
\end{abstract}

Results: Duplex RNA were designed to have incomplete complementarity with the 3'UTR sequences of both target genes. One such duplex, CODEMIR-I, was found to suppress VEGF and ICAM-I by 90 and $60 \%$, respectively in ARPE- 19 cells at a transfected concentration of $40 \mathrm{ng} / \mathrm{mL}$. Use of a cyan fusion reporter with target sites constructed in its 3'UTR demonstrated that the repression of VEGF and ICAM-I by CODEMIR-I was indeed due to interaction with the target sequence. An exhaustive analysis of sequence variants of CODEMIR-I demonstrated a clear positive correlation between activity against VEGF (but not ICAM-I) and the length of the contiguous complementary region (from the $5^{\prime}$ end of the guide strand). Various strategies, including the use of inosine bases at the sites of divergence of the target sequences were investigated.

Conclusion: Our work demonstrates the possibility of designing multitargeting dsRNA to suppress more than one disease-altering gene. This warrants further investigation as a possible therapeutic approach.

\section{Background}

The different triggers eliciting RNAi all ultimately lead to the formation of short ( 21 nucleotide) RNA duplexes termed short interfering RNAs (siRNAs). Complete complementarity between the guide strand and the target
mRNA leads to catalytic cleavage of the mRNA and suppresses gene expression [1]. Endogenous microRNAs (miRNAs) are also small duplex RNAs with diverse and critical roles in gene regulation [2]. miRNAs share many of the features of siRNAs including the loading of the guide 
strand into a RNA induced silencing complex (RISC) [3]. In contrast to siRNAs, mammalian miRNAs do not generally exhibit high complementarity to their cognate target sites. Binding of miRNAs to their target sites may induce target degradation or may prevent translation and reduce gene expression at the protein level [4]. In mammals, miRNAs are thought to bind to partially complementary sites predominantly located in the 3 ' untranslated regions (UTRs) of target mRNAs $[2,4,5]$, thereby enabling the coordinate regulation of genes containing such sites.

Whilst the factors affecting siRNA activity have been extensively studied $[3,6-8]$, the parameters affecting miRNA-mediated translational suppression have not yet been definitively elucidated. Binding of the $5^{\prime}$ end of the guide strand to the target mRNA appears to be critical, with an almost absolute requirement for complementarity at the so-called "seed site" from positions 2-7 (measuring from the 5' end of the guide strand) [9-11]. miRNA target sites appear to be almost exclusively located in the 3 'UTRs of target genes $[11,12]$, and miRNA target sites may be functionally restricted to 3' UTRs, since binding of miRNAs to other sites in the transcript does not induce translational suppression.

The present study demonstrates proof of concept for the design of artificial short RNAs with at least partial complementarity to multiple unrelated transcripts, and which suppress the expression of the corresponding unrelated genes. The interfering RNAs described herein were designed to target expression of VEGF-A and ICAM-1, two genes involved in ocular neovascular disease [13]. We found that for suppression of VEGF-A, the length of complementarity to the seed region, as well as the total complementarity of the guide strand to the target were important determinants of activity. This relationship was not observed for ICAM-1, however this discrepancy appeared to result from specific sequence motifs in those guide strands with high ICAM-1 complementarity. Thus, the length of seed complementarity, and overall complementarity between the guide strand and the target should be considered in the design of multitarget interfering RNAs.

\section{Results}

Transcript sequences corresponding to the 3' UTRs of VEGF-A and ICAM-1 (ensembl IDs ENST00000356655 and ENST00000264832, respectively) were used to search for a suitable seed of at least 6 contiguous bases present in both genes. A pool of all possible seeds of 6 bases or greater was generated using the specified length as a window and advancing the window in a stepwise fashion 1 base at a time. Low complexity seeds were eliminated and the pool was further restricted to those for which at least 3 contiguous bases were predicted to bind to an unpaired region in at least $50 \%$ of optimal and suboptimal (within $-1 \mathrm{kcal} / \mathrm{mol}$ of optimal) folded structures (as determined using the Vienna RNA package [14]).

Two different seeds were selected for experimental testing: one of 12 bases in length and one of 7 bases that was within a genetic context that favoured the design of "consensus target sequences" (comprising the seed sequence and a consensus of the sequence adjacent to the seed sequence from the desired targets, in this instance, VEGF$A$ and ICAM-1). Multiple consensus target sequences were generated for both seeds by first aligning the target sequences (VEGF-A and ICAM-1) relative to their shared seed sequences. Then, extensions 5 ' to the seed sequences were proposed to a length of 21 nucleotides. The exact complements of these 21 nucleotide consensus target sequences were assessed in silico for hybridisation to the target sequences, and one sequence for each seed was selected for experimental testing. "Passenger" strands were designed to be complementary to the selected "guide" strands over a duplex length of $19 \mathrm{nt}$ with 3' overhangs of 2 nucleotides (UU added in the case of the passenger strand).

Annealed RNA duplexes or single-stranded RNA oligonucleotides were purchased from Sigma-Proligo. Where required, $100 \mu \mathrm{M}$ oligonucleotides were annealed in 50 $\mu \mathrm{M}$ Tris, $100 \mathrm{mM} \mathrm{NaCl}$ by heating to $90^{\circ} \mathrm{C}$ and cooling to $4^{\circ} \mathrm{C}$ over 3-4 hours. Control siRNA sequences are shown in Additional file 1.

Because of the complexity of human disease, we sought to determine if it was possible to develop novel interfering RNA that can multi-target one or more pre-selected therapeutic targets. We achieved this by identifying short regions of homology in pre-selected target RNA with bioinformatic techniques. These "seeds" were then used to design short duplex RNAs having one strand that binds with at least partial complementarity to each target RNA sequence. Examples of such COmputationally DEsigned Multi-targeting Interfering RNAs (CODEMIRs) were sought to simultaneously target two genes (VEGF-A and ICAM-1) that are associated with ocular neovascular disease [13]. Two candidate seeds (7 and 12 contiguous bases) that are present in the 3' UTRs of both target mRNA were identified and used to design "consensus target sequences" (see Methods and Fig. 1a). The sequences complementary to these consensus target sequences (corresponding to the theoretical guide strands of CODEMIRs) were evaluated for binding to the target mRNAs using RNAhybrid software [15]. Two of these guide strands, one targeting each seed, were chosen for experimental testing; each having strong predicted binding to both target genes (Figs. 1b, c). 
a

$$
\begin{aligned}
& \text { a } \\
& \text { VEGF-A }{ }^{5} \text { GGGAUUCCUGUAGACACACCCACCCACAUA } \\
& \text { ICAM-1 ACCUUUGUUAGCCACCUCCCACCCACAUA }
\end{aligned}
$$

$\begin{array}{ll} & \text { UAGAGACACCCCACCCACAUA } \\ \text { Consensus } & \text { UUAGACCACCCCACCCACAUA } \\ \text { target sequences } & \text { AGACACCACCCCACCCACAUA } \\ & \text { UUAGACUCACCCACCCACAUA } \\ & \text { AGACACUCACCCACCCACAUA }\end{array}$

b CODEMIR-1

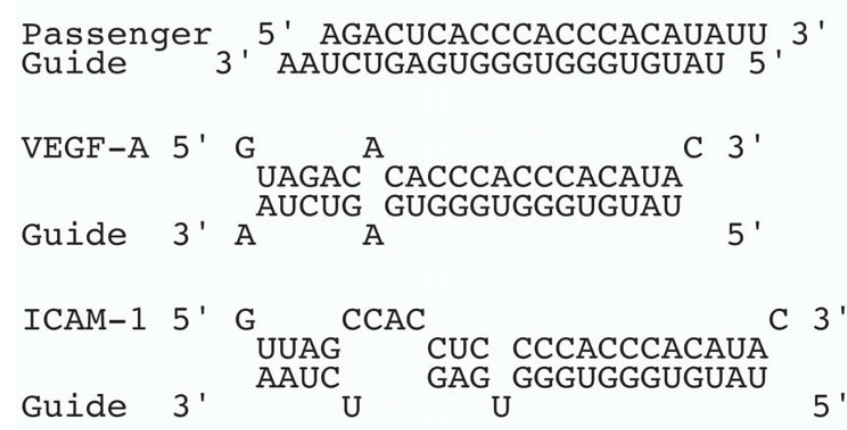

C

\section{CODEMIR-2}

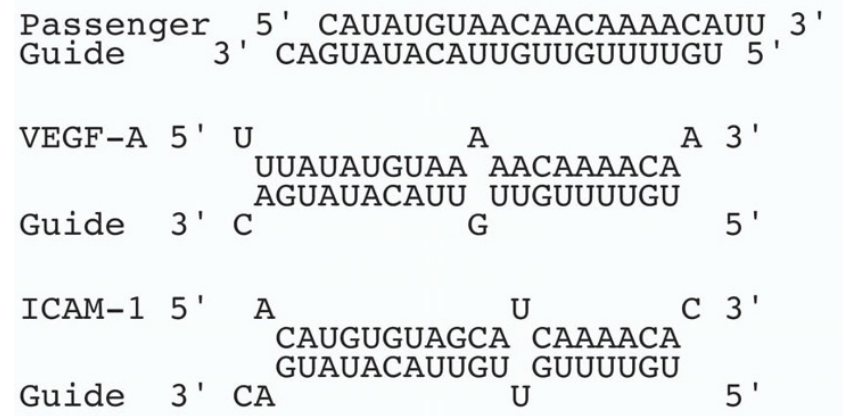

Figure I

Design of CODEMIRs targeting VEGF-A and ICAM-I.

(a) Example of seed site alignment and consensus target sequence design for VEGF-A and ICAM-I using a I 2 nucleotide seed. (b) and (c) Schematic illustration of CODEMIR-I and -2 (respectively) and guide strand binding to the VEGF-A and ICAM-I mRNAs. Top strand represents the target mRNA ( $5^{\prime}$ to $\left.3^{\prime}\right)$, bottom strand indicates the guide strand ( $3^{\prime}$ to $\left.5^{\prime}\right)$.

Transfection of these CODEMIRs into the retinal epithelial cell line ARPE-19 induced significant suppression of both VEGF-A and ICAM-1 (Fig. 2a), demonstrating the feasibility of designing synthetic RNAs that suppress the expression of more than one gene at the protein level. The effects were qualitatively similar whether stimulation with deferoxamine or IL- $1 \beta$ was used or cells were cultured without stimulation. However, stimulation afforded a much greater dynamic range for the measurement of both VEGF-A and ICAM-1 enabling improved discrimination between CODEMIR designs. Thus, stimulation conditions were used in all experiments reported herein. CODEMIR1 (which demonstrated greater efficacy than CODEMIR2) was further characterised. CODEMIR-1 demonstrated dose-dependent suppression of VEGF-A and ICAM-1 expression in ARPE-19 cells (Fig. 2b). In contrast, the irrelevant cytokine IL-8, as measured by ELISA of culture supernatant (R\&D Systems) was not affected by CODEMIR-1 under similar conditions, demonstrating the specificity of the effect. In order to confirm that seed binding was required for the activity of CODEMIR-1 against VEGF-A and ICAM-1, nucleotide substitutions introduced at positions 4,4 and 6, or 4, 6 and 8 of the guide strand of CODEMIR-1 were tested in ARPE cells (Additional file 1). With the exception of CODEMIR-122 (position 4 mismatch), these had significantly impaired suppression of both VEGF-A and ICAM-1 relative to CODEMIR-1 at the protein level (Fig. 2c). Moreover, expression of a cyan fluorescent reporter gene containing the CODEMIR-1 target site in the 3'UTR was suppressed by CODEMIR-1 in a manner that correlated well with the observed suppression of endogenous VEGF-A and ICAM-1 (Fig. 2d), demonstrating that the observed suppression of VEGF-A and $I C A M-1$ was mediated by the binding of the guide strand of CODEMIR-1 to its predicted target sites. The above results all relate to expression at the protein level, however, at the mRNA level, CODEMIR-1 also caused significant suppression of both VEGF-A and ICAM-1, and this suppression was reduced by mismatches in the seed region (Fig. 2e).

Because the CODEMIR-1 target site appeared particularly amenable to suppression, we focused on variants of this CODEMIR. In a systematic approach, 32 variants of CODEMIR-1 were designed by developing a consensus sequence for the VEGF-A and ICAM-1 target sites and alternating between the VEGF-A and ICAM-1 complementary base at mismatched positions (Additional files 1 and 2) with the exception of mismatches that could be accommodated for by wobble base pairing with the eventual guide strand (eg G binding to either C or U). All 32 of these CODEMIRs were tested for the ability to suppress both endogenous VEGF-A and ICAM-1 (Fig. 3) and a cyan fluorescent protein reporter (Additional file 2) in cell culture. Suppression of the endogenous genes correlated well with suppression of the reporter (Additional file 2), particularly for VEGF-A where the range of suppression was greater. For VEGF-A, there was a clear positive correlation between activity and the length of the contiguous complementary region (from the 5 ' end of the guide strand) and 

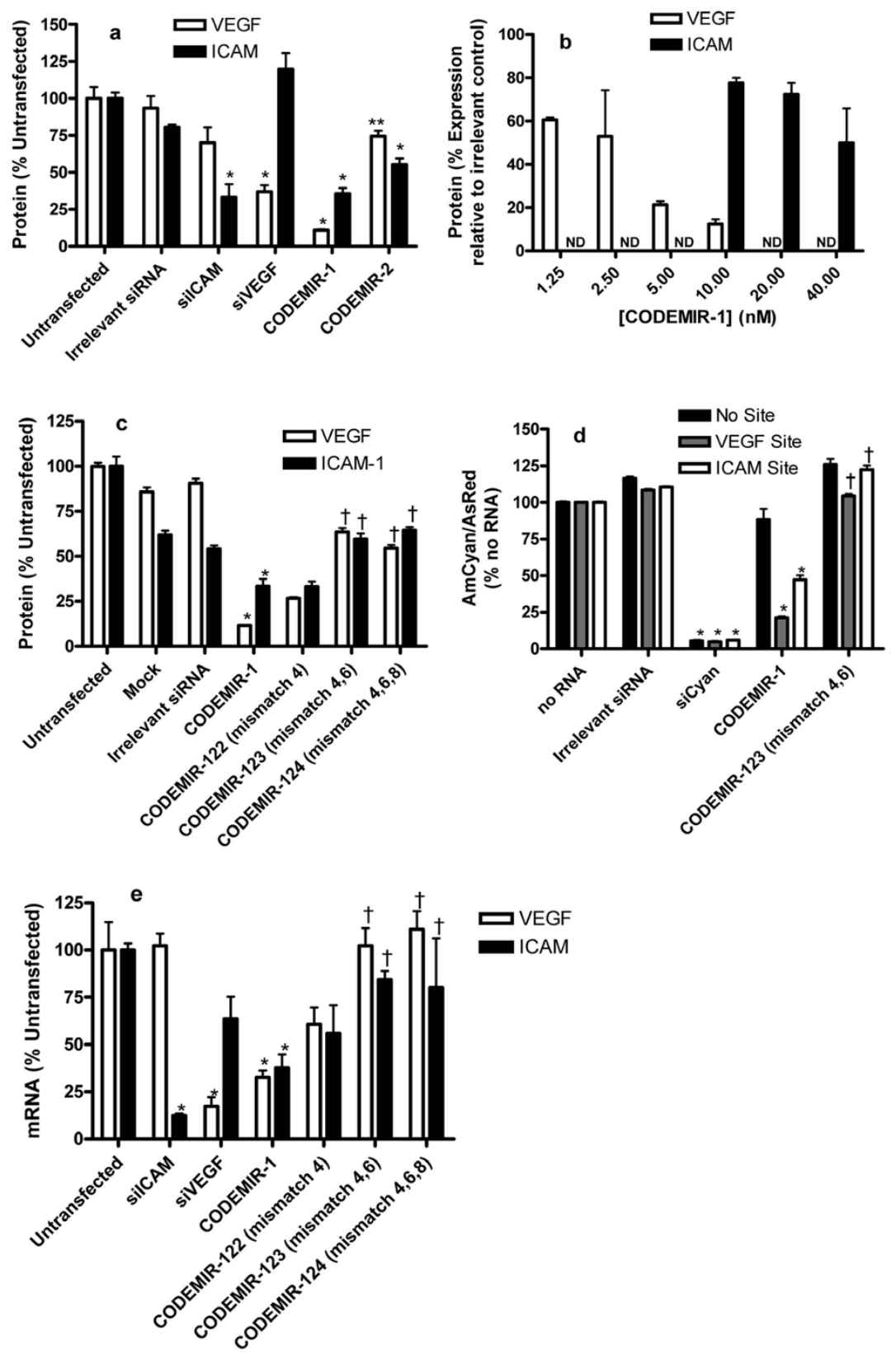

Figure 2

Suppression of VEGF-A and ICAM-I expression by CODEMIR-I and -2. Unless otherwise indicated, VEGF-A (ELISA) or ICAM-I (FACS) were assayed in ARPE- 19 cells 48 hours post-transfection and 24 hours post-stimulation with I $30 \mu M$ Deferoxamine or I ng/mL IL-I $\beta$ respectively. All data points indicate the mean of triplicate samples. Error bars show standard deviation. Statistical significance was determined by two-way ANOVA using a Bonferroni post-test $(* p<0.001$ as compared to either untransfected and/or irrelevant siRNA; ** $p<0.00 \mathrm{I}$ as compared to untransfected and $p<0.01$ as compared to irrelevant siRNA; $\dagger p<0.00$ I compared to CODEMIR-I). (a) Gene suppression by CODEMIR-I and -2 . (b) Dose responsiveness of gene suppression by CODEMIR-I. Cells were transfected at the indicated concentrations. (ND = not determined). (c) Effect of mismatches on gene suppression by CODEMIR-I. (d) Suppression of VEGF-A and ICAM-I reporter constructs by CODEMIRI ARPE-I 9 cells were co-transfected with the AmCyan reporter and AsRed control plasmids and 40 nM indicated RNA duplexes. Fluorescence was assessed by FACS 48 hours post-transfection. (e) Suppression of VEGF-A and ICAM-I mRNA expression by selected CODEMIRs. For VEGF-A, stimulation was performed 24 hours post-transfection using $65 \mu M$ Deferoxamine and the QuantiGene ${ }^{\circledR}$ assay was performed on cell lysates prepared 24 hours post-stimulation. For ICAM-I the QuantiGene ${ }^{\circledR}$ assay was performed on cell lysates prepared 24 hours post-transfection. 
a clear negative correlation between activity and the number of mismatches between the target and the CODEMIR (Additional file 2). By contrast, there was no such correlation between ICAM-1 suppression and the length of the contiguous stretch of complementarity and the correlation between activity and the number of mismatches between the target and the CODEMIR was, in this case, reversed (Additional file 2). Indeed, the variant (CODEMIR-64) which was completely complementary to the ICAM-1 mRNA exhibited very poor activity against ICAM-1. However, those CODEMIRs with high complementarity to the ICAM-1 mRNA all contained a region of at least 5 contiguous $G$ nucleotides, a feature that is known to be detrimental to siRNA activity [8]. Substitution of the $\mathrm{G}$ at position 14 of the guide strand with an $\mathrm{A}$ in CODEMIR-56 and CODEMIR-76 (replacing the predicted G:U wobble base with an A:U base pair; Additional file 1) significantly improved the suppressive activity of these CODEMIRs against both the endogenous and reporter genes (Fig. $4 \mathrm{a}, \mathrm{b}$ ); confirming that runs of $\mathrm{G}$ nucleotides impair CODEMIR activity.

As confirmation that the length of 5' complementarity between CODEMIR-1 and the VEGF-A mRNA was critical to suppressive activity, we compared the activity of an interfering RNA complementary to the CODEMIR-1 VEGF-A target site (siVAIC) with 5 variants containing introduced mismatches at positions 9, 10, 11 and/or 12 of the guide strand (Additional file 1 and Fig. 5a). Mismatches at any of these positions reduced suppressive activity from $>90 \%$ to $50-60 \%$. This suggested that these central mismatches abrogated RISC-mediated cleavage of the mRNA, but that translational suppression, causing the retained moderate reduction in VEGF-A protein levels, was less dependent upon complementarity at these central positions. This was confirmed by semi-quantitative RT-PCR detection of the VEGF-A mRNA (Fig. 5b) which demonstrated reduction of VEGF-A transcript level with an siRNA (siVAIC) directed to the CODEMIR-1 site, but not with any of the central mismatch containing variants.

Since the length of $5^{\prime}$ complementarity appeared to be relevant to activity on VEGF-A, we investigated CODEMIRs which contained inosine bases (which are capable of base pairing with all 4 naturally occurring ribobases, albeit with varying affinities) at crucial points (sites that cannot be matched to all transcripts and are near the RISC cleavage site). Three variants of CODEMIR-1 were designed which included inosine bases at positions 13 and/or 15 of the guide strand (CODEMIRs 100-102; Additional file 1). These CODEMIRs showed comparable ICAM-1 suppressive activity to CODEMIR-1 (which contains a mismatch at position 13), but reduced VEGF-A suppression relative to CODEMIR-1 in the case of CODEMIR-100 and CODEMIR-102 (Fig. 6a). The comparable activity against
ICAM-1 may simply reflect the fact that translational repression against ICAM-1 is largely dependent upon the seed binding alone, and so is not affected by alterations in the 3 ' tail. The VEGF-A suppressive activity of inosine-containing variants of CODEMIR-1 was also compared to similar variants of CODEMIR-1 with mismatches to the VEGF-A mRNA at positions 13 and/or 15 (CODEMIRs 68-71; Additional file 1). None of the inosine-containing variants demonstrated substantially improved activity compared to the corresponding mismatched variant (Fig. $6 \mathrm{~b})$. This indicates that inosine base pairs, although tolerated in some positions, may not necessarily be useful in overcoming mismatches between guide strand and target, although our analysis may not be generalizable given that the binding of dI to each base varies and is contextdependent.

There are 3 bases common to both ICAM-1 and VEGF-A immediately downstream (3') of the CODEMIR-1 target site. We investigated whether increasing the length of the complementary region (to both targets) would increase activity. Three variants of CODEMIR-1 were designed such that the guide strand sequence was shifted 1-3 bases 5 ' of the CODEMIR-1 sequence (Additional file 1). However, none of these were as active as CODEMIR-1 against either target (Fig. 7). Whilst the variants shifted 1 and 2 bases (CODEMIRs 11 and 12) would be predicted to have compromised strand loading due to the introduction of a $\mathrm{G}$ :C base pair close to the $5^{\prime}$ end of the guide strand, the variant shifted 3 bases (CODEMIR-13) would be predicted to retain a strong loading bias towards the guide strand.

Mammalian miRNAs have generally been shown to bind in the 3' UTRs of target genes, and the CODEMIRs described above all targeted 3' UTRs. To assess whether the targeting of $3^{\prime}$ UTR regions is advantageous when there is incomplete complementarity between the target and the guide strand of a duplex RNA in the central region (known to be required for Ago-2 cleavage in the case of siRNA) dsRNA duplexes were designed such that they were completely complementary to the VEGF-A mRNA, excepting the nucleotides at positions 10 and 11 of the guide strand (Additional file 1). To minimize variations in efficacy due to sequence composition or strand-loading bias, each of these duplexes contained a similar bulge composition (CU) and was relatively GC rich at the 3 ' end of the guide strand. Of these 16, 3 targeted the VEGF-A 5' UTR, 5 targeted the VEGF-A ORF and 8 targeted the VEGFA 3'UTR. In general, the duplexes targeting regions in the 5' UTR or ORF displayed poor VEGF-A suppression, whereas those targeting regions in the 3' UTR displayed strong suppression (Fig. 8a). Moreover, there was a significant difference in activity between the duplexes targeting the ORF and those targeting the 3'UTR ( $\mathrm{p}<0.01$; Fig. $8 \mathrm{~b}$ ). 


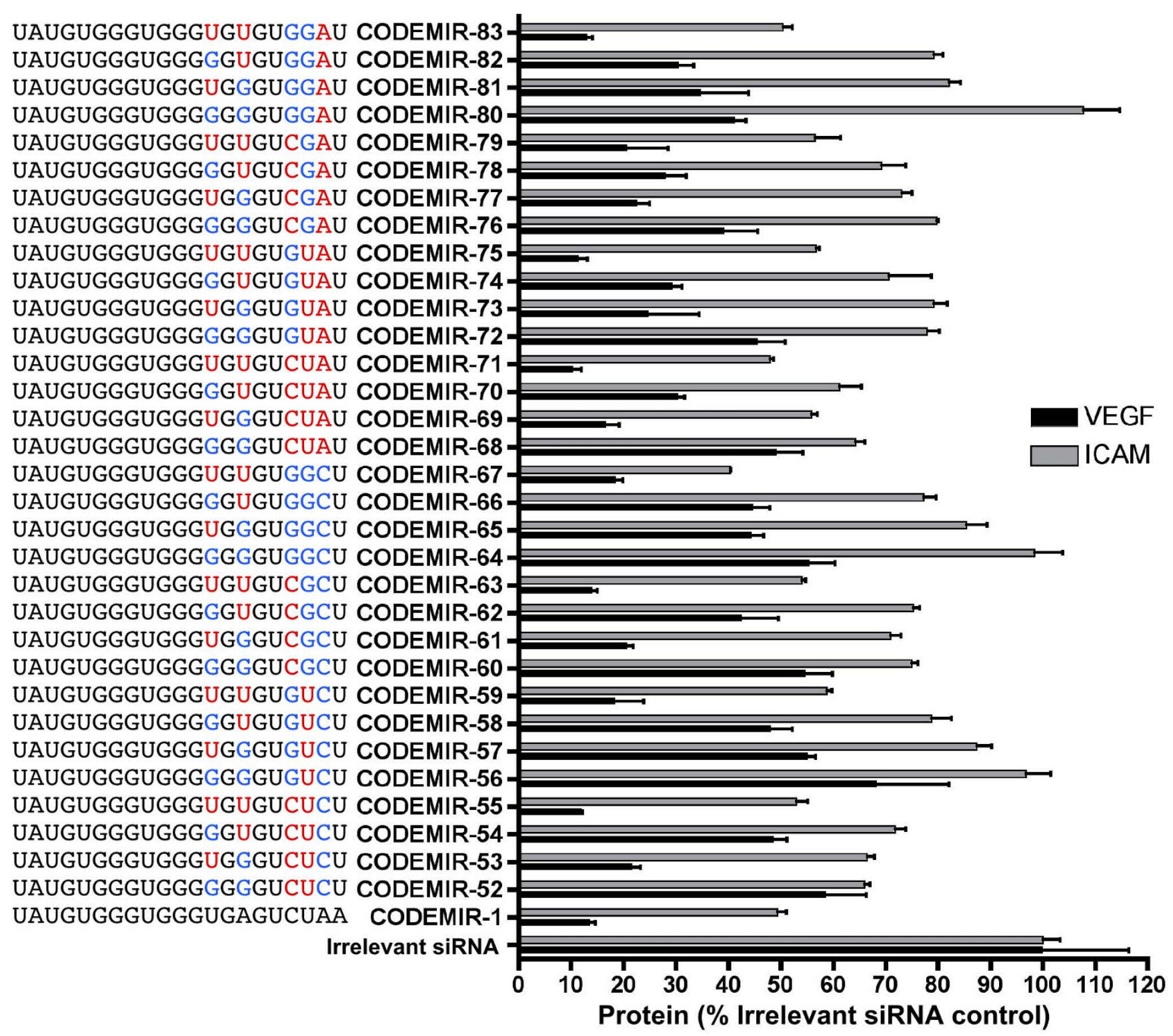

Figure 3

VEGF-A and ICAM-I suppressive activity of 32 variants of CODEMIR-I. ARPE- 19 cells were transfected with $40 \mathrm{nM}$ of the indicated RNA duplex, and VEGF-A (ELISA) and ICAM-I (FACS) were assayed 48 hours post-transfection and 24 hours after stimulation with $130 \mu$ M Deferoxamine or I $\mathrm{ng} / \mathrm{mL}$ IL-I $\beta$ respectively. The guide strand of each CODEMIR is shown in the $5^{\prime}$ to $3^{\prime}$ direction, with blue bases indicating mismatches to the VEGF-A target sequence and red bases indicating mismatches to the ICAM-I target sequence. Data points represent the mean of triplicate samples. Error bars indicate standard deviation.

This demonstrates that the 3' UTR of VEGF- $A$ mRNA is, relative to other regions of the mRNA, more suited to suppression through miRNA-like translational repression, which in part is likely to account for the high activity of CODEMIR-1 and its variants against this target.

The use of RNA duplexes is potentially confounded by offtarget inflammatory cellular responses [16]. To confirm the specificity of action of CODEMIR-1 we examined the expression of IFN $\beta$ and STAT1. After transfection with
CODEMIR-1, no evidence of up-regulation of either of these genes was observed (Additional file 2). In a recent separate study to be reported elsewhere, we studied the activation of TLR7/8 by 207 siRNA sequences when transfected with DOTAP into fresh human PBMCs at concentrations up to $100 \mathrm{nM}$. CODEMIR-1 belonged to the least active subset of sequences (with $\mathrm{IC}_{50}>100 \mathrm{nM}$ ), confirming low propensity of this sequence for activation of RNAsensing innate receptors. 

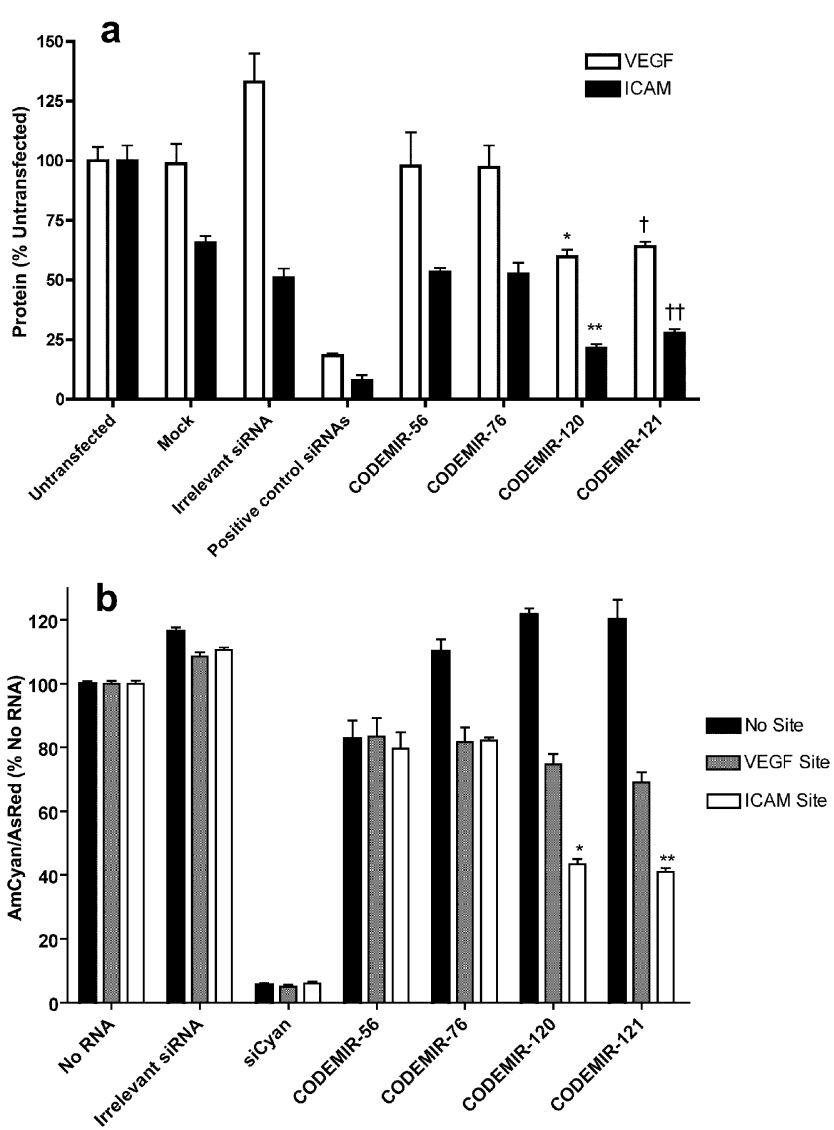

Figure 4

Comparison of CODEMIR-I variants with (\#56 and \#76) and without (\#I 20 and \#I 2 I) 7 G motifs. (a) ARPE- 19 cells were transfected with $40 \mathrm{nM}$ duplex RNA and VEGF (ELISA) or ICAM (FACS) were assayed 48 hours posttransfection $\left({ }^{*} p<0.001\right.$, ${ }^{*} *_{p}<0.01$ as compared to CODEMIR-56; $\uparrow p<0.01$, $\uparrow \dagger p<0.05$ as compared to CODEMIR-76). (b) Normalised fluorescence of ARPE- 19 cells transfected with AmCyan/CODEMIR-I reporter and AsRed plasmids and CODEMIR variants with and without 7 $G$ motifs. ARPE- 19 cells were co-transfected with I $\mu \mathrm{g}$ of the plasmids and $40 \mathrm{nM}$ indicated RNA duplexes. Fluorescence was assessed by FACS 48 hours post-transfection (*p $<0.00$ I as compared to CODEMIR-56, ${ }^{*} p<0.001$ as compared to CODEMIR-76). Each bar represents the mean of triplicate samples. Error bars indicate standard deviation. Statistical significance was determined by two-way ANOVA using a Bonferroni post-test.

\section{Discussion}

The present study demonstrates proof of concept for the approach of using short interfering RNAs with at least partial complementarity to two target transcripts for suppression of the expression of unrelated genes. Although we have only presented data for two seed sequences in two genes here, we have successfully used the same approach to target unrelated genes implicated in diverse disease
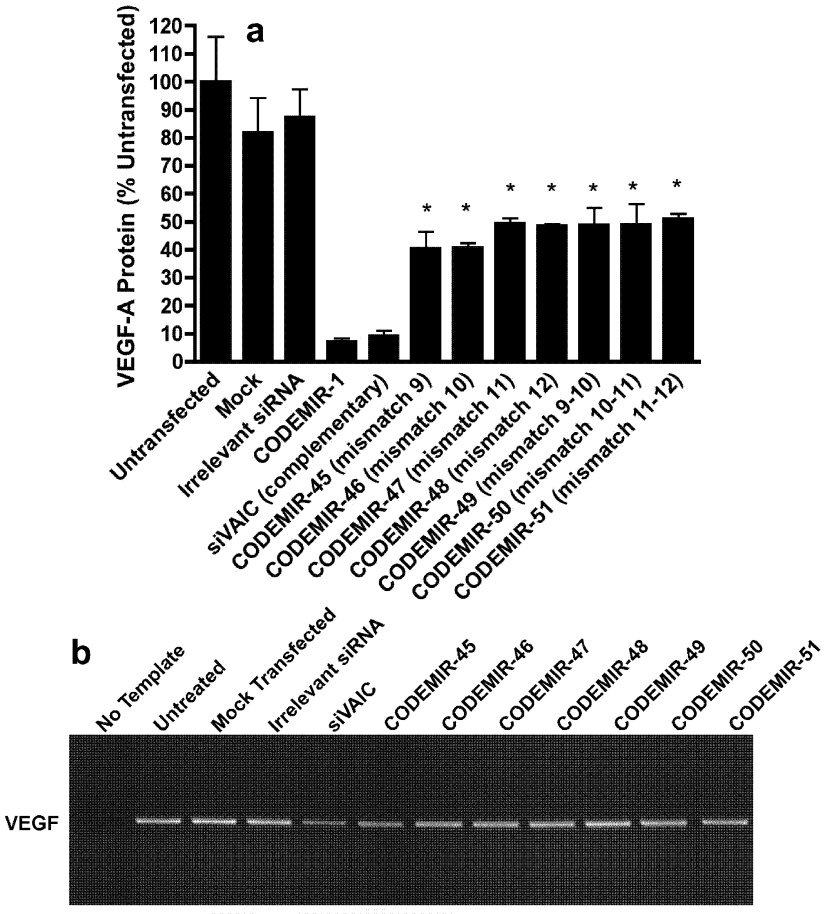

GAPDH

Figure 5

Central mismatches at the CODEMIR-I target site impair VEGF-A suppressive activity. (a) ARPE- I 9 cells were transfected with $40 \mathrm{nM}$ of the indicated RNA duplex, and VEGF-A secretion was measured by ELISA 48 hours post-transfection ( $*_{p}<0.00$ I by ANOVA as compared to CODEMIR-I). All data points represent the mean of triplicate samples. Error bars indicate standard deviation. (b) VEGF mRNA expression in ARPE-19 cells transfected with centrally mismatched variants of CODEMIR-I. Semi-quantitative RT-PCR performed on RNA from ARPE- 19 cells transfected with $40 \mathrm{nM}$ indicated RNA duplexes demonstrated impaired degradation of VEGF-A mRNA by CODEMIRs mismatched relative to a perfectly complementary control (siVAIC). Gel is representative result from duplicate experiments.

states including oncology, virology and inflammation [17]. Thus, this approach can be used as a general technique for the suppression of multiple genes using a single interfering RNA.

Current pharmaceutical research is dominated by a reductionist "one-disease one-target" paradigm. However, the complex nature of many diseases has increasingly led to the realisation that activity against multiple targets may be required for effective treatment [18]. Practically, this can be achieved through the use of multiple agents, and a number of combination drugs have recently become available for the treatment of heart disease and HIV infection, amongst 

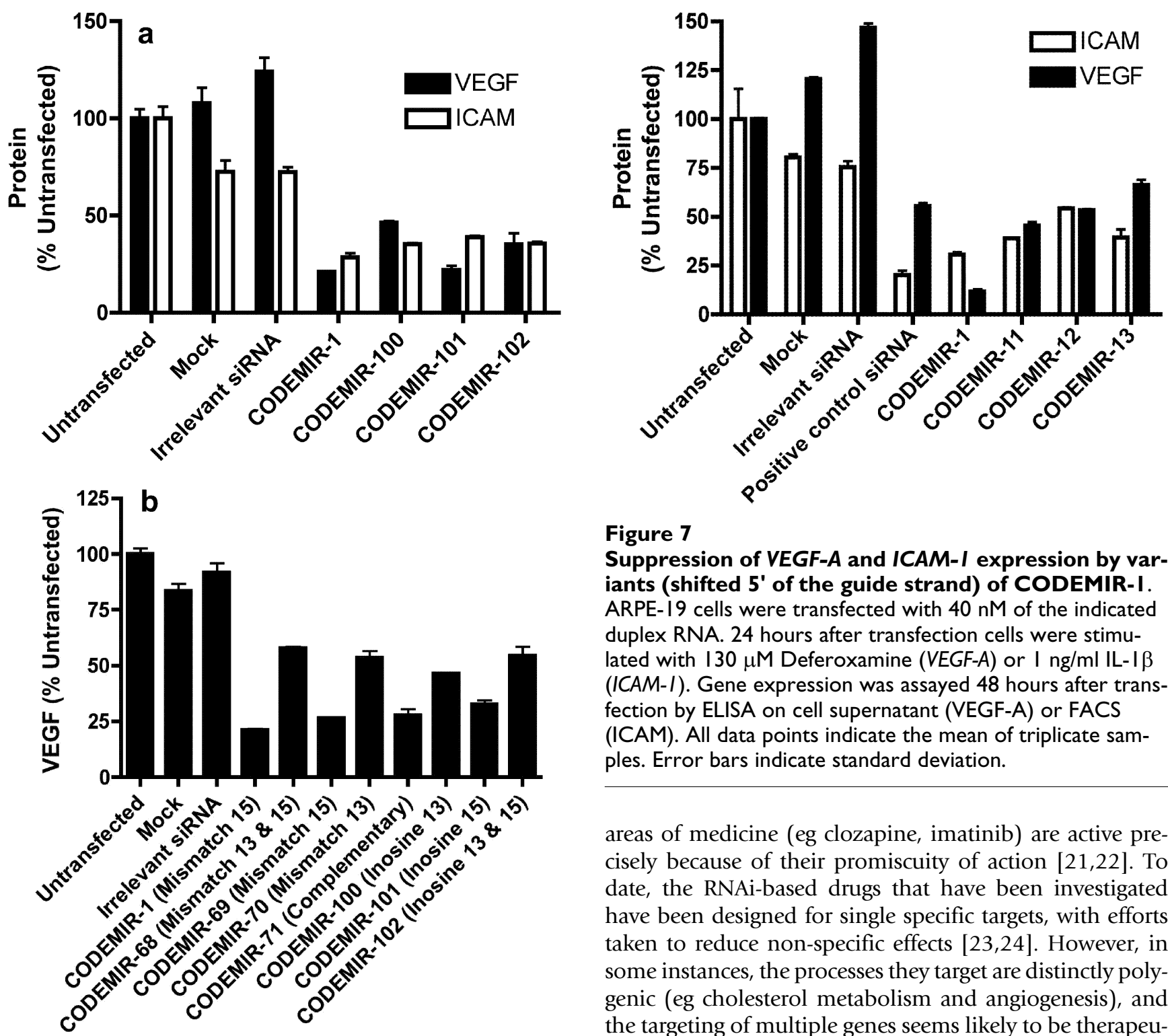

Figure 6

(a) VEGF and ICAM expression in ARPE- 19 cells after transfection with inosine containing CODEMIRs (\#100-102). ARPE-19 cells were transfected with $40 \mathrm{nM}$ duplex RNA and VEGF-A (ELISA) or ICAM-I (FACS) were assayed 48 hours post-transfection. (b) Comparison of VEGF$A$ suppressive activity of CODEMIRs containing inosine bases or mismatches at positions 13 and/or 15 of the guide strand. ARPE- 19 cells were transfected with $10 \mathrm{nM}$ duplex RNA and VEGF-A (ELISA) was assayed 48 hours post-transfection. Each bar represents the mean of triplicate samples. Error bars indicate standard deviation.

other indications. However, the combination of multiple agents can lead to unforseen interactions [19]. Alternatively, agents with activity against multiple targets can be developed [20]. Indeed, many successful drugs in different

Figure 7

Suppression of VEGF-A and ICAM- I expression by variants (shifted 5 ' of the guide strand) of CODEMIR-I. ARPE-1 9 cells were transfected with $40 \mathrm{nM}$ of the indicated duplex RNA. 24 hours after transfection cells were stimulated with $130 \mu$ M Deferoxamine (VEGF-A) or I ng/ml IL-I $\beta$ (ICAM-I). Gene expression was assayed 48 hours after transfection by ELISA on cell supernatant (VEGF-A) or FACS (ICAM). All data points indicate the mean of triplicate samples. Error bars indicate standard deviation.

areas of medicine (eg clozapine, imatinib) are active precisely because of their promiscuity of action $[21,22]$. To date, the RNAi-based drugs that have been investigated have been designed for single specific targets, with efforts taken to reduce non-specific effects $[23,24]$. However, in some instances, the processes they target are distinctly polygenic (eg cholesterol metabolism and angiogenesis), and the targeting of multiple genes seems likely to be therapeutically beneficial. Whilst the targeting of multiple genes could be achieved through the use of a mixture of active siRNAs, we believe that using a single active has a number of possible advantages. First, having a single active reduces the complexity of clinical and product development. Secondly, mixtures of siRNA can have disappointing effects because of competition for the RISC machinery [25]. Thirdly, a single active may have reduced off-target effects relative to a pool of actives, since each siRNA has a unique pattern of off-target effects and a mixture may thus increase the magnitude and/or scope of off-target effects.

Our finding that the length of seed complementarity affects CODEMIR activity independent of overall complementarity is surprising given the current understanding of microRNA-target interactions $[4,9,11]$. Mismatches to the target in the central positions of the CODEMIR-1 guide 

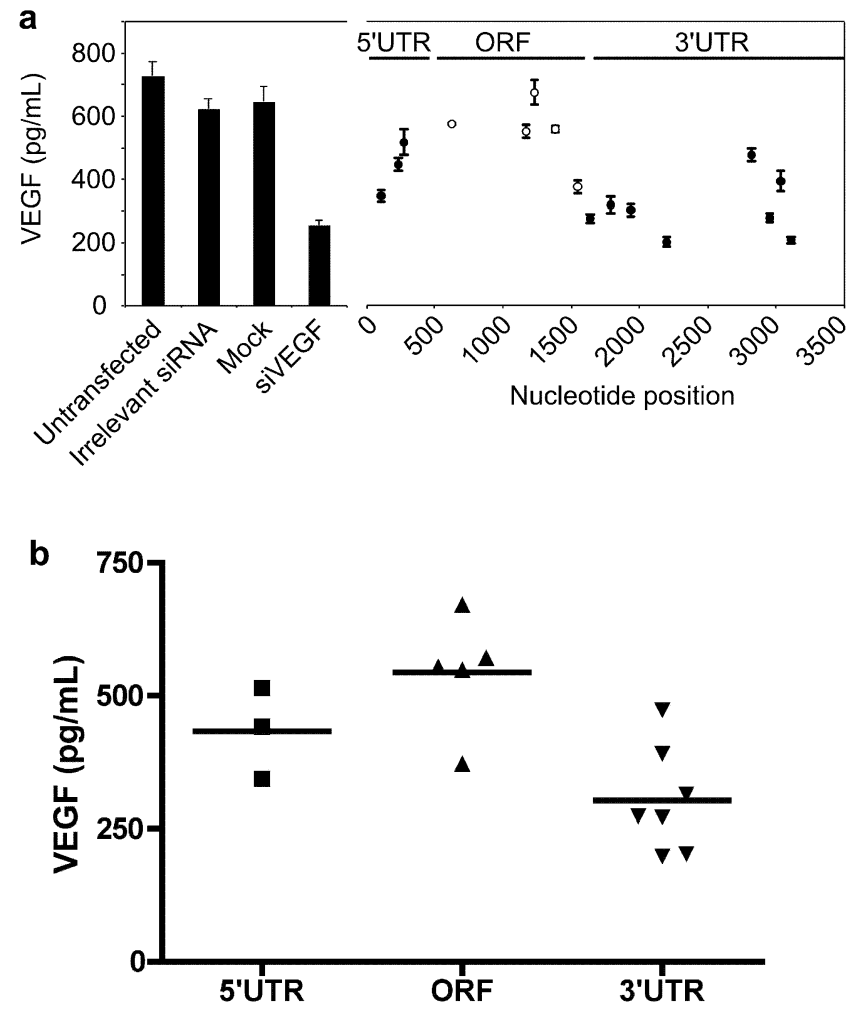

Figure 8

Analysis of target site selection. (a) VEGF-A suppression by 2 I nt RNA duplexes (with central mismatches) targeting different regions of the VEGF-A mRNA. ARPE- 19 cells were transfected with $40 \mathrm{nM}$ indicated RNA duplexes. VEGF-A in cell culture supernatant was measured by ELISA 48 hours post-transfection and 24 hours post-stimulation with I $30 \mu \mathrm{M}$ Deferoxamine. Each point represents the mean of triplicate samples. Error bars indicate standard deviation. (b) Statistical analysis of data for VEGF-A suppression depicted in Panel (a). There was a significant difference $(p<0.05)$ between the means for molecules targeting the $3^{\prime} U T R$ as compared to molecules targeting the ORF as determined by one-way ANOVA with a Bonferroni post-test.

strand decreased VEGF-A suppression at the protein level to $50-60 \%$, and largely abrogated suppression at the RNA level. This suggests that complementarity to the target at the 5 '-end and in the centre of the guide strand is sufficient to induce target cleavage, and that CODEMIRs with central mismatches to the targets may act primarily through translational repression. As such, CODEMIRs with longer 5 ' regions of contiguous complementarity may exhibit higher efficacy. However, suppression of multiple genes related to the same phenotype is likely to have significant advantages over targeting a single gene, and thus CODEMIRs with central mismatches will still be of significant utility.

\section{Conclusion}

The present study validates the approach of using a short dsRNA molecule specifically designed with a single guide strand to suppress the expression of multiple unrelated genes implicated in a particular medical condition. We have shown that synthetic duplex RNAs with at least partial complementarity to multiple transcripts are capable of specific suppression of multiple target genes. Given the multi-genic nature of many disease states, such multi-targeting interfering RNAs may offer significant therapeutic benefits relative to single target siRNAs. Our findings also have relevance to the biology of miRNA-target interactions, particularly with respect to the effect of unpaired bases on miRNA-mediated suppression of translation.

\section{Methods \\ Cell culture and transfection}

ARPE-19 cells were cultured in Dulbecco's Modified Eagle's Medium supplemented with 10\% Fetal bovine serum and $10 \mathrm{mM}$ glutamine (Gibco). Duplex RNA transfection was performed using Lipofectamine 2000 (Invitrogen), at a ratio of $260 \mathrm{ng}$ siRNA per $1 \mu \mathrm{L}$ Lipofectamine, according to the manufacturer's instructions. Cells were transfected 24 hours after seeding at a density of 1.25 $10^{4}$ cells per $\mathrm{cm}^{2}$ in 96,48 or 24 -well plates. RNA duplex and plasmid co-transfection was performed with $1 \mu \mathrm{g}$ reporter plasmid (AmCyan based - see below), $1 \mu \mathrm{g}$ control plasmid (pAsRed-C1 - Clontech) and 40 pmol RNA duplex together complexed with $10 \mu \mathrm{g}$ Lipofectamine 2000 and applied to cells in a 12 well plate.

\section{ELISA and FACS analysis of gene expression}

VEGF-A concentrations in cell supernatants were assayed 48 hours after transfection and 24 hours after stimulation with $130 \mu \mathrm{M}$ Deferoxamine (Sigma) using a commercially available ELISA kit (R\&D Systems) according to the manufacturer's instructions. Cell surface ICAM-1 was assayed by flow cytometry. Cells seeded into 12 well plates were transfected with RNA duplexes 24 hours after seeding. ICAM-1 expression was assayed 48 hours after transfection and 24 hours after stimulation with $1 \mathrm{ng} / \mathrm{mL}$ recombinant human Interleukin-1 $\beta$ (R\&D systems): cells were trypsinized, stained with $0.5 \mu \mathrm{g}$ anti-human ICAM-1 mouse $\operatorname{IgG}_{1}$ antibody (Becton Dickinson) at $4^{\circ} \mathrm{C}$ for 20 minutes, washed with PBS, stained with $0.2 \mu \mathrm{g}$ Phycoerythrin-labelled anti-mouse $\operatorname{IgG}_{1}$ antibody (Becton Dickinson) at $4{ }^{\circ} \mathrm{C}$ for 20 minutes, washed with PBS and analysed using a FACScalibur flow cytometer (Becton Dickinson). IFN $\beta$ production was assayed using a commercially available ELISA (InVitrogen) according to the manufacturer's instructions. For detection of fluorescent reporter expression, mean fluorescence in the reporter channel (FL-1) was normalised to mean fluorescence in 
the control channel (FL-2). Statistical analysis was performed using Prism software (GraphPad Software Inc.)

\section{RNA quantification}

For semi-quantitative RT-PCR, total cellular RNA was extracted using the RNeasy kit (Qiagen) according to the manufacturer's instructions. Reverse transcription was performed on $1 \mu \mathrm{g}$ total RNA using a commercially available kit (First-Strand cDNA Synthesis Kit, Marligen Biosciences). PCR of GAPDH was used to standardise the amounts of starting cDNA. PCR was performed in $1 \times$ PCR buffer II (Applied Biosystems) supplemented with 3.75 $\mathrm{mM} \mathrm{MgCl} 2,250 \mu \mathrm{M}$ each dNTP, $250 \mathrm{nM}$ each primer and $0.125 \mathrm{U} / \mu \mathrm{L}$ AmpliTaq gold (Applied Biosystems). VEGF$A$ was amplified by 30 cycles of $95^{\circ} \mathrm{C}-30$ seconds, $60^{\circ} \mathrm{C}$ - 30 seconds and $72^{\circ} \mathrm{C}-30$ seconds, using the primers (5' to 3 '): TTC TTG CTG CTA AAT CAC CGA and GAA CAT TCC CCT CCC AAC TCA. GAPDH was amplified by 18 cycles of $95^{\circ} \mathrm{C}-30$ seconds, $65^{\circ} \mathrm{C}-30$ seconds and $72^{\circ} \mathrm{C}$ - 30 seconds, using the primers ( $5^{\prime}$ to $3^{\prime}$ ): CTG CTT CAC CAC CTT CTT GAT GTC ATC ATA and GAC CCC TTC ATT GAC CTC AAC TAC ATG GT.

VEGF-A, ICAM-1, IFN $\beta$, STAT1 and GAPDH RNA were quantified using a Quantigene ${ }^{\circledast}$ branched DNA assay (Panomics), according to the manufacturer's instructions.

\section{Plasmid construction}

Fluorescent reporter vectors were constructed by cloning target sites into the 3'UTR of the AmCyan1 fluorescent protein gene in the pAmCyan1-C1 vector (Clontech). A stop codon was inserted by cloning the duplex oligodeoxynucleotide pair GAT CTC TCG AGT GAT AGG and AAT TCC TAT CAC TCG AGA into the BglII and EcoRI sites of pAmCyan1-C1. Specific CODEMIR-1 target site reporters were generated by cloning the duplex oligonucleotide pairs AAT TTC CTG TAG ACA CAC CCA CCC ACA TAC and GAT CGT ATG TGG GTG GGT GTG TCT ACA GGA (VEGF-A), and AAT TTG TTA GCC ACC TCC CCA CCC ACA TAC and GAT CGT ATG TGG GTG GGG AGG TGG CTA ACA (ICAM-1) into the EcoRI and BamHI sites of the stop codon-containing pAmCyan1-C1 vector. The fulllength VEGF-A 3'UTR (including stop codon) was cloned from ARPE-19 cells by RT-PCR using the primers GGG CTC GAG TGA GCC GGG CAG GAG G (Forward) and GGG GTC GAC TAC GGA ATA TCT CGA AAA ACT (Reverse) and cloned into the XhoI and SalI sites of the pAmCyan1-C1.

\section{Western blot analysis}

Total protein lysates were obtained by lysing cells in RIPA buffer $(150 \mathrm{mM} \mathrm{NaCl}, 0.1 \%$ sodium dodecyl sulphate, $1 \%$ nonidet P-40, $0.5 \%$ sodium deoxycholate, $50 \mathrm{mM}$ Tris-Cl, $\mathrm{pH} 8$ ). Protein concentrations were determined by the Lowry protocol using the Bio-Rad $D_{C}$ Protein Assay.
Total proteins $(10 \mu \mathrm{g})$ were separated by electrophoresis on NuPage $4-12 \%$ Bis-Tris gels (Invitrogen) and transferred onto nitrocellulose membranes. Membranes were blocked with 3\% BSA in TBST (10 mM Tris pH 8, $30 \mathrm{mM}$ $\mathrm{NaCl}, 0.05 \%$ Tween) for $20 \mathrm{~min}$ at room temperature. After rinsing with TBST twice, anti-STAT1 mouse IgG $_{1}$ (1:400; Santa Cruz Biotechnology) and anti- $\beta$-actin mouse $\operatorname{IgG}_{1}$ (1:2000; Sigma-Aldrich) in TBST-MLK (TBST containing $5 \%$ dried skim milk) were added and incubated for 1 hour at room temperature. After 3 - $5 \mathrm{~min}$ washes in TBST, membranes were incubated with horseradish peroxidase conjugated anti-mouse Ig (1:2000 in TBS-MLK; Dako Cytomation) for $45 \mathrm{~min}$ at room temperature. The membrane was washed in TBST ( $3-5 \mathrm{~min})$ and analysed by chemiluminescence using ECL Western blotting detection (Amersham).

\section{Authors' contributions}

TP performed in vitro cell assays, ELISAs, FACS analysis, molecular cloning and drafted the manuscript. MMG carried out the immune stimulation assays. AG performed in vitro cell assays and ELISAs. AK performed the RT-PCR. MP performed the bioinformatic analyses. GMA and DJB conceived of, and participated in the design of the study. LPR conceived of, and participated in the design of the study and helped draft the manuscript. All authors read and approved the final manuscript.

\section{Additional material}

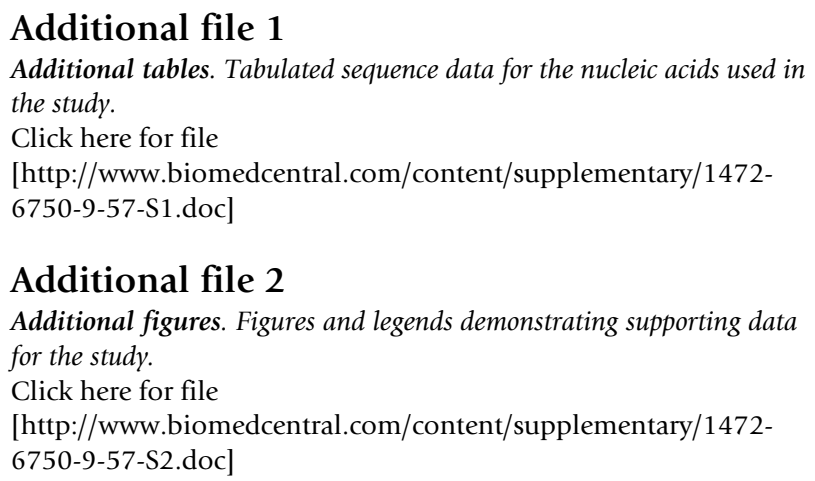

\section{Additional file 2}

Additional figures. Figures and legends demonstrating supporting data for the study.

Click here for file

[http://www.biomedcentral.com/content/supplementary/1472-

6750-9-57-S2.doc]

\section{Acknowledgements}

The study was funded and performed with the support of Johnson and Johnson Research Pty Limited.

\section{References}

I. Schwarz DS, Ding H, Kennington L, Moore JT, Schelter J, Burchard J, Linsley PS, Aronin N, Xu Z, Zamore PD: Designing siRNA That Distinguish between Genes That Differ by a Single Nucleotide. PLoS Genet 2006, 2(9):

2. Kloosterman WP, Plasterk RH: The diverse functions of microRNAs in animal development and disease. Dev Cell 2006, I I(4):44I-450. 
3. Khvorova A, Reynolds A, Jayasena SD: Functional siRNAs and miRNAs exhibit strand bias. Cell 2003, II5(2):209-2I6.

4. Engels BM, Hutvagner G: Principles and effects of microRNAmediated post-transcriptional gene regulation. Oncogene 2006, 25(46):6163-6169.

5. Filipowicz W, Jaskiewicz L, Kolb FA, Pillai RS: Post-transcriptional gene silencing by siRNAs and miRNAs. Curr Opin Struct Biol 2005, I 5(3):33I-34I.

6. Schwarz DS, Hutvagner G, Du T, Xu Z, Aronin N, Zamore PD: Asymmetry in the assembly of the RNAi enzyme complex. Cell 2003, I I5(2): 199-208.

7. Martinez J, Patkaniowska A, Urlaub H, Luhrmann R, Tuschl T: Singlestranded antisense siRNAs guide target RNA cleavage in RNAi. Cell 2002, I I 0(5):563-574.

8. Reynolds A, Leake D, Boese Q, Scaringe S, Marshall WS, Khvorova A: Rational siRNA design for RNA interference. Nat Biotechnol 2004, 22(3):326-330.

9. Brennecke J, Stark A, Russell RB, Cohen SM: Principles of microRNA-target recognition. PLoS Biol 2005, 3(3):e85.

10. Doench JG, Sharp PA: Specificity of microRNA target selection in translational repression. Genes Dev 2004, 1 8(5):504-5 I I.

II. Grimson A, Farh KK, Johnston WK, Garrett-Engele P, Lim LP, Bartel DP: MicroRNA targeting specificity in mammals: determinants beyond seed pairing. Mol Cell 2007, 27(I):9I- 105.

12. Lai EC: Micro RNAs are complementary to 3' UTR sequence motifs that mediate negative post-transcriptional regulation. Nat Genet 2002, 30(4):363-364.

13. Funatsu H, Yamashita H, Sakata K, Noma H, Mimura T, Suzuki M, Eguchi S, Hori S: Vitreous levels of vascular endothelial growth factor and intercellular adhesion molecule $I$ are related to diabetic macular edema. Ophthalmology 2005, I I 2(5):806-8I6.

14. Hofacker IL: Vienna RNA secondary structure server. Nucleic Acids Res 2003, 3 I(13):3429-3431.

15. Rehmsmeier M, Steffen P, Hochsmann M, Giegerich R: Fast and effective prediction of microRNA/target duplexes. Rna 2004, I0(10): $1507-1517$.

16. Marques JT, Devosse $T$, Wang $D$, Zamanian-Daryoush $M$ Serbinowski P, Hartmann R, Fujita T, Behlke MA, Williams BR: A structural basis for discriminating between self and nonself double-stranded RNAs in mammalian cells. Nat Biotechnol 2006, 24(5):559-565.

17. Rivory LRPM, Birkett DJ, Arndt GM, Passioura TJ: Multitargeting Interfering RNAs and Methods of their Use and Design. International Patent Application: WO2007/056826AI.

18. Imming $P$, Sinning $C$, Meyer A: Drugs, their targets and the nature and number of drug targets. Nat Rev Drug Discov 2006, 5(I0):82I-834.

19. Frantz S: The trouble with making combination drugs. Nat Rev Drug Discov 2006, 5(I I):88I-882.

20. Morphy $R$, Rankovic $Z$ : The physicochemical challenges of designing multiple ligands. I Med Chem 2006, 49( I 6):496 I-4970.

21. Meltzer HY: An overview of the mechanism of action of clozapine. J Clin Psychiatry 1994, 55(Suppl B):47-52.

22. Pardanani A, Tefferi A: Imatinib targets other than bcr/abl and their clinical relevance in myeloid disorders. Blood 2004, 104(7): 1931-1939.

23. Jackson AL, Burchard J, Schelter J, Chau BN, Cleary M, Lim L, Linsley PS: Widespread siRNA "off-target" transcript silencing mediated by seed region sequence complementarity. Rna 2006 | 2(7): | | 179-| | 87.

24. Zimmermann TS, Lee AC, Akinc A, Bramlage B, Bumcrot D, Fedoruk MN, Harborth J, Heyes JA, Jeffs LB, John M, et al.: RNAi-mediated gene silencing in non-human primates. Nature 2006 , 44 I(7089): | ||-|| |4.

25. Castanotto D, Sakurai K, Lingeman R, Li H, Shively L, Aagaard L Soifer H, Gatignol A, Riggs A, Rossi J]: Combinatorial delivery of small interfering RNAs reduces RNAi efficacy by selective incorporation into RISC. Nucleic Acids Res 2007, 35(I5):5154-5164.
Publish with Biomed Central and every scientist can read your work free of charge

"BioMed Central will be the most significant development for disseminating the results of biomedical research in our lifetime. "

Sir Paul Nurse, Cancer Research UK

Your research papers will be:

- available free of charge to the entire biomedical community

- peer reviewed and published immediately upon acceptance

- cited in PubMed and archived on PubMed Central

- yours - you keep the copyright

Submit your manuscript here:

http://www.biomedcentral.com/info/publishing_adv.asp
BioMedcentral 